\title{
Finite Element Solution of One Dimensional Flow in Unsaturated Porous Media
}

\author{
N.S.Rabari ${ }^{1}$, A.S.Gor ${ }^{2}$, P.H. Bhathawala ${ }^{3}$ \\ ${ }^{1}$ Research Scholar, Department of Mathematics, H.N.G.University, Patan-384265, Gujarat, India \\ ${ }^{2}$ Principal, Pramukh Swami Science College, Kadi-382715, Gujarat, India \\ ${ }^{3}$ Professor of IT, AURO University, Surat - 394510, Gujarat, India
}

\begin{abstract}
In the present paper we have discuss finite element method for the partial differential equation governing one dimensional unsteady flow through unsaturated porous media. Finite element method is a numerical method for finding an approximate solution of differential equation in finite region or domain. In FEM, we generate difference equation by using approximate method such as Weighted residual method, Variational method with piecewise polynomial solution.
\end{abstract}

Keywords: Finite Element Method, Unsteady Flow, Unsaturated Porous Media, Variational Method

\section{Introduction}

The unsteady and unsaturated flow of water through soils is due to content changes as a function of time and the entire pore spaces are not completely filled with flowing liquid respectively. Knowledge concerning such flows helps some workers like hydrologist, agriculturalists, many fields of science and engineering. The water infiltrations system and the underground disposal of seepage and waste water are encountered by these flows, which are described by nonlinear partial differential equation.

The mathematical model conforms to the hydrological situation of one dimensional vertical ground water recharge by spreading [17]. Such flow is of great importance in water resource science, soil engineering and agricultural sciences. In the present paper, we have obtained a numerical solution of the problem by finite element techniques using MATLAB.

\section{Statement Of The Problem}

In the investigated mathematical model, we consider that the groundwater recharge takes place over a large basin of such geological location that the sides are limited by rigid boundaries and the bottom by a thick layer of water table. In this case, the flow is assumed vertically downwards through unsaturated porous media.

It is assumed that the diffusivity coefficient is equivalent to its average value over the whole range of moisture content, and the permeability of the media is continuous linear function of the moisture content. The theoretical formulation of the problem yields a nonlinear partial differential equation for the moisture content.

\section{Mathematical Formulation Of The Problem}

The equation of continuity for an unsaturated medium is given by

$$
\frac{\partial}{\partial t}\left(\rho_{s} \theta\right)=-\nabla \cdot M
$$

Where, $\rho_{s}$ is the bulk density of the medium, $\theta$ is its moisture content on a dry weight basis and $\mathrm{M}$ is the mass flux of moisture.

From Darcy's law $[15,16,17]$ for the motion of water in a porous medium, we get,

$$
V=-k \nabla \phi
$$

Where, $\nabla \phi$ represents the gradient of the whole moisture potential, $\mathrm{V}$ the volume flux of moisture, and $\mathrm{k}$ the coefficient of aqueous conductivity. Combining equations (1) and (2) we obtain,

$$
\frac{\partial}{\partial t}\left(\rho_{s} \theta\right)=\nabla \cdot(\rho k \nabla \phi)
$$

Where, $\rho$ is the fluid density. Since, in the present case, we consider that the flow takes place only in the vertical direction, equation (3) reduces to,

$$
\rho_{s} \frac{\partial \theta}{\partial t}=\frac{\partial}{\partial z}\left(\rho K \frac{\partial \psi}{\partial z}\right)-\frac{\partial}{\partial z}(\rho K g)
$$


Where, $\psi$ the capillary pressure potential, $\mathrm{g}$ is the gravitational constant and $\phi=\psi-g z \quad[19-23]$. The positive direction of the z-axis is the same as that of the gravity. Considering $\theta$ and $\psi$ to be connected by a single valued function, we may write (4) as,

$$
\frac{\partial \theta}{\partial t}=\frac{\partial}{\partial z}\left(D \frac{\partial \theta}{\partial z}\right)-\frac{\rho}{\rho_{S}} g \frac{\partial K}{\partial z}
$$

Where, $\mathrm{D}=\frac{\rho}{\rho_{S}} k \frac{\partial \psi}{\partial \theta}$ and is called diffusivity coefficient.

Replacing D by its average value $\mathrm{D}_{\mathrm{a}}$ and assuming $\mathrm{K}=\mathrm{K}_{0} \theta, \mathrm{K}_{0}=0.232$, we have

$$
\frac{\partial \theta}{\partial t}=D_{a} \frac{\partial^{2} \theta}{\partial z^{2}}-\frac{\rho}{\rho_{s}} K_{0} \frac{\partial \theta}{\partial z}
$$

Considering the water table to be situated at a depth L, and putting:

$$
\frac{z}{L}=\xi, \quad \frac{t D_{a}}{L^{2}}=T, \quad \beta_{0}=\frac{\rho}{\rho_{s}} \frac{K_{0}}{D_{a}}
$$

We may write the boundary value problem as:

$$
\frac{\partial \theta}{\partial T}=\frac{\partial^{2} \theta}{\partial \xi^{2}}-\beta_{0} \frac{\partial \theta}{\partial \xi}
$$

$\xi \quad=$ Penetration depth (dimensionless)

$\mathrm{T} \quad=$ time (dimensionless)

$\beta_{0} \quad=$ Flow parameter $\left(\mathrm{cm}^{2}\right)$

$\rho=$ Mass density of water $(\mathrm{gm})$

$\rho_{s} \quad=$ Bulk density of the medium on dry weight basis $\left(\mathrm{gmcm}^{-3}\right)$

$\mathrm{K}_{0} \quad=$ Slope of the permeability vs moisture content plot $\left(\mathrm{cmsec}^{-1}\right)$

$\mathrm{D}_{\mathrm{a}} \quad=$ Average value of the diffusivity coefficient over the whole range of moisture content $\left(\mathrm{cm}^{2} \mathrm{sec}^{-1}\right)$

It may be mentioned for definiteness that a set of appropriate boundary conditions are

$$
\begin{gathered}
\theta(0, T)=\theta_{0}, \quad \frac{\partial \theta}{\partial \xi}(1, T)=0 \\
\theta(\xi, 0)=0
\end{gathered}
$$

Where the moisture content throughout the region is zero initially, at the layer $\mathrm{z}=0$ it is $\theta_{0}$, and at the water table $(\mathrm{z}=\mathrm{L})$ it is assumed to remain $100 \%$ throughout the process of investigation. It may be remarked that the effect of capillary action at the stationary groundwater level, being small, is neglected.

The following values of the various parameters have been considered in the analysis: $\beta=\mathbf{b}=\mathbf{2 . 0 3 5}, \boldsymbol{\theta}_{\mathbf{0}}=\mathbf{0 . 5}$, A MATLAB code is prepared and executed with $\mathbf{h}=\mathbf{1 / 1 5}$ and $\mathbf{k}=\mathbf{0 . 0 0 2 2 2 3}$ for $\mathbf{2 2 5}$ time levels. The numerical value are shown by table. Curves indicating the behavior of moisture content corresponding to various time period have been shown in corresponding figures .

\section{Finite Element Solution}

The domain of the problem, in present case, consists of all points between $\xi=0$ and $\xi=1$ (Figure 1(a)). This domain is divided in to set of linear elements (Figure 1(b)).

$$
\xi=0 \quad \xi=1
$$

Figure 1(a) 


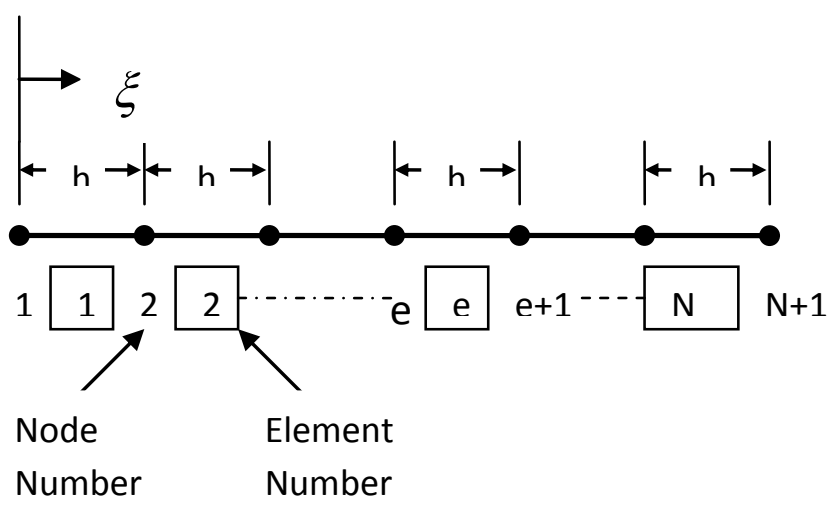

Figure 1(b)

Now, the variational form of given Partial Differential equation is

$$
J(\theta)=\frac{1}{2} \int_{R}\left[\left(\frac{\partial \theta}{\partial \xi}\right)^{2}+2 \theta \frac{\partial \theta}{\partial T}-\beta \theta \frac{\partial \theta}{\partial \xi}\right] d \xi
$$

Choose an arbitrary linear element $R^{(\mathrm{e})}=\left[\theta_{1}{ }^{(\mathrm{e})}, \theta_{2}{ }^{(\mathrm{e})}\right]$ \& obtain interpolation function for $\mathrm{R}^{(\mathrm{e})}$ using Lagrange interpolation Method such as

$$
\begin{aligned}
& \theta^{(e)}(\xi)=\sum_{j=1}^{2} N_{j}(\xi) \theta_{j}^{(e)}=N^{(e)} \phi^{(e)}=\phi^{(e)^{T}} N^{(e)^{T}} \\
& \text { where } \quad N^{(e)}=\left[\begin{array}{llll}
N_{1} & N_{2}
\end{array}\right] \quad \& \quad \phi^{(e)}=\left[\begin{array}{ll}
\theta_{1} & \theta_{2}
\end{array}\right]^{T}
\end{aligned}
$$

For linear element, Shape function is

$$
\begin{aligned}
& N_{1}=\frac{\xi-\xi_{1}}{\xi_{2}-\xi_{1}} \text { and } N_{2}=\frac{\xi_{2}-\xi}{\xi_{2}-\xi_{1}} \\
& \text { э } \quad N_{1}(\xi)+N_{2}(\xi)=1 \\
& N_{j}\left(\xi_{i}\right)= \begin{cases}1 & j=i \\
0 & j \neq i\end{cases}
\end{aligned}
$$

Now, apply Variational Method to $\mathrm{R}^{(\mathrm{e})}$, therefore equation (10) becomes,

$$
J\left(\theta^{(e)}\right)=\frac{1}{2} \int_{R^{(e)}}\left[\left(\frac{\partial \theta^{(e)}}{\partial \xi}\right)^{2}+2 \theta^{(e)} \frac{\partial \theta^{(e)}}{\partial T}-\beta \theta^{(e)} \frac{\partial \theta^{(e)}}{\partial \xi}\right] d \xi
$$

As, $\quad \theta^{(e)}(\xi)=N^{(e)} \phi^{(e)}=\phi^{(e)^{T}} N^{(e)^{T}}$

$$
\therefore \quad \frac{\partial \theta^{(e)}}{\partial \xi}=\frac{\partial N^{(e)}}{\partial \xi} \phi^{(e)}=\phi^{(e)^{T}} \frac{\partial N^{(e)^{T}}}{\partial \xi}
$$

$$
\text { and }\left(\frac{\partial \theta^{(e)}}{\partial \xi}\right)^{2}=\phi^{(e)^{T}} \frac{\partial N^{(e)^{T}}}{\partial \xi} \frac{\partial N^{(e)}}{\partial \xi} \phi^{(e)}
$$

Therefore, equation (13) becomes,

$$
J\left(\theta^{(e)}\right)=\frac{1}{2} \int_{R^{(e)}} \phi^{(e)^{T}}\left[\left(\frac{\partial N^{(e)^{T}}}{\partial \xi} \frac{\partial N^{(e)}}{\partial \xi}\right) \phi^{(e)}+2\left(N^{(e)^{T}} N^{(e)}\right) \frac{\partial \phi^{(e)}}{\partial T}-\beta\left(\frac{\partial N^{(e)^{T}}}{\partial \xi} N^{(e)}\right) \phi^{(e)}\right] d \xi
$$


For minimization, first differentiate equation (14) with respect to $\phi^{(e)}$

$\frac{\partial J^{(e)}}{\partial \phi^{(e)}}=\int_{R^{(e)}}\left[\left(\frac{\partial N^{(e)^{T}}}{\partial \xi} \frac{\partial N^{(e)}}{\partial \xi}\right) \phi^{(e)}+2\left(N^{(e)^{T}} N^{(e)}\right) \frac{\partial \phi^{(e)}}{\partial T}-\beta\left(\frac{\partial N^{(e)^{T}}}{\partial \xi} N^{(e)}\right) \phi^{(e)}\right] d \xi$

Now, $\frac{\partial J^{(e)}}{\partial \phi^{(e)}}=0$.

Therefore the element equation is,

$$
A^{(e)} \frac{\partial \phi^{(e)}}{\partial T}+B^{(e)} \phi^{(e)}+C^{(e)} \phi^{(e)}=0
$$

Where,

$$
\mathrm{A}^{(e)}=\int_{\theta_{1}^{(e)}}^{\theta_{2}^{(e)}}\left(N^{(e)^{T}} N^{(e)}\right) d \xi, \mathrm{B}^{(e)}=-\beta \int_{\theta_{1}^{(e)}}^{\theta_{2}^{(e)}}\left(\frac{\partial N^{(e)^{T}}}{\partial \xi} N^{(e)}\right) d \xi, \mathrm{C}^{(e)}=\int_{\theta_{1}^{(e)}}^{\theta_{2}^{(e)}}\left(\frac{\partial N^{(e)^{T}}}{\partial \xi} \frac{\partial N^{(e)}}{\partial \xi}\right) d \xi_{I}
$$

We use Gauss Legendre Quadrature Method to evaluate these integral. For this, first we transform co-ordinate $\xi$ to a local coordinate z $\ni \quad$ when $\xi=\theta_{1}, z=-1 ; \quad \xi=\theta_{2}, z=1$.

Therefore interpolation function becomes $\quad N_{1}(z)=\frac{1}{2}(1-z) ; N_{2}(z)=\frac{1}{2}(1+z)$ and Jacobian matrix is $J=\frac{1}{2} h$

Also element matrix transform to

$$
\begin{aligned}
& \mathrm{A}^{(e)}=\int_{-1}^{1}\left(N^{(e)^{T}} N^{(e)}\right) J d z \approx \sum_{I=1}^{r} A^{(e)}\left(z_{I}\right) W_{I} \\
& B^{(e)}=-\beta \int_{-1}^{1}\left(\frac{1}{J} \frac{\partial N^{(e)^{T}}}{\partial z} N^{(e)}\right) J d z \approx \sum_{I=1}^{r} B^{(e)}\left(z_{I}\right) W_{I} \\
& C^{(e)}=\int_{-1}^{1}\left(\frac{1}{J} \frac{\partial N^{(e)^{T}}}{\partial z} \frac{1}{J} \frac{\partial N^{(e)}}{\partial z}\right) J d z \approx \sum_{I=1}^{r} C^{(e)}\left(z_{I}\right) W_{I}
\end{aligned}
$$

For $\mathrm{A}^{(\mathrm{e})}$, degree of polynomial $\mathrm{p}=2$ then $\mathrm{r}=2$ and For $\mathrm{B}^{(\mathrm{e})}$ and $\mathrm{C}^{(\mathrm{e})}, \mathrm{p}=1$ then $\mathrm{r}=1 . \mathrm{z}_{\mathrm{I}}$ and $\mathrm{W}_{\mathrm{I}}$ are corresponding gauss points and gauss weights with respect to ' $\mathrm{r}$ '. Then, the element matrix becomes,

$$
\mathbf{A}^{(e)}=\frac{h^{(e)}}{6}\left[\begin{array}{ll}
2 & 1 \\
1 & 2
\end{array}\right], \quad \mathbf{B}^{(e)}=\frac{-\beta}{2}\left[\begin{array}{cc}
-1 & -1 \\
1 & 1
\end{array}\right], \quad C^{(e)}=\frac{1}{h^{(e)}}\left[\begin{array}{cc}
1 & -1 \\
-1 & 1
\end{array}\right]
$$

\subsection{Assembling Of Elements}

In deriving the element equations, we isolated a typical element (the eth element) from the mesh and formulated the variational problem and developed its finite element model. To obtain the finite element equations of the total problem, we must put the elements back into their original positions. The assembly of linear elements is carried out by imposing the following two conditions:

a) The continuity of primary variable requires

$$
\theta_{n}^{e}=\theta_{1}^{e+1}
$$

b) The balance of secondary variables at connecting nodes requires

$$
\theta_{n}^{e}+\theta_{1}^{e+1}=\left\{\begin{array}{l}
0 \quad \text { if no external po int surceis applied } \\
\theta_{1} \quad \text { if anexternal point surce of magnitude } \theta_{1} \text { is applied }
\end{array}\right.
$$


The inter-element continuity of primary variable can be imposed by simply renaming the variables of all elements connected to common node. For example, for a mesh of $\mathrm{N}$ linear finite element connected in series, we have

$$
\theta_{1}^{1}=\vartheta_{1}, \quad \theta_{2}^{1}=\theta_{1}^{2}=\vartheta_{2}, \quad \theta_{2}^{2}=\theta_{1}^{3}=\vartheta_{3}, \cdots \cdots, \theta_{2}^{N-1}=\theta_{1}^{N}=\vartheta_{N}, \quad \theta_{2}^{N}=\vartheta_{N+1}
$$

For a uniform mesh of $\mathrm{N}$ elements, by eq. (15) \& eq. (16), the assembled equation becomes

$$
A \frac{\partial \varphi}{\partial T}-B \phi+C \phi=0
$$

Where,

$$
\begin{aligned}
& \mathrm{A}=\frac{h}{6}\left[\begin{array}{ccccccc}
2 & 1 & 0 & 0 & \ldots & 0 & 0 \\
1 & (2+2) & 1 & 0 & \ldots & 0 & 0 \\
0 & 1 & (2+2) & 0 & \ldots & 0 & 0 \\
\ldots & \ldots & \ldots & \ldots & \ldots & \ldots & \ldots \\
\ldots & \ldots & \ldots & \ldots & \ldots & \ldots & \ldots \\
0 & 0 & 0 & 0 & \ldots & (2+2) & 1 \\
0 & 0 & 0 & 0 & \ldots & 1 & 2
\end{array}\right], \mathrm{B}=\frac{-\beta}{2}\left[\begin{array}{ccccccc}
-1 & -1 & 0 & 0 & \ldots & 0 & 0 \\
1 & (1-1) & -1 & 0 & \ldots & 0 & 0 \\
0 & 1 & (1-1) & 0 & \ldots & 0 & 0 \\
\ldots & \ldots & \ldots & \ldots & \ldots & \ldots & \ldots \\
\ldots & \ldots & \ldots & \ldots & \ldots & \ldots & \ldots \\
0 & 0 & 0 & 0 & \ldots & (1-1) & -1 \\
0 & 0 & 0 & 0 & \ldots & 1 & 1
\end{array}\right] \\
& \mathrm{C}=\frac{1}{h}\left[\begin{array}{ccccccc}
1 & -1 & 0 & 0 & \ldots & 0 & 0 \\
-1 & (1+1) & -1 & 0 & \ldots & 0 & 0 \\
0 & -1 & (1+1) & 0 & \ldots & 0 & 0 \\
\ldots & \ldots & \ldots & \ldots & \ldots & \ldots & \ldots \\
\ldots & \ldots & \ldots & \ldots & \ldots & \ldots & \ldots \\
0 & 0 & 0 & 0 & \ldots & (1+1) & -1 \\
0 & 0 & 0 & 0 & \ldots & -1 & 1
\end{array}\right] \phi=\left[\begin{array}{llllll}
\theta_{1} & \theta_{2} & \ldots & \ldots & \theta_{N+1}
\end{array}\right] \\
&
\end{aligned}
$$

Equation (17) represents the assembled equation.

\subsection{Time Approximation}

We have obtained the finite element equation in the global form, which represent a system of simultaneous ordinary differential equation. We now introduced $\delta$ family of approximations which approximates weighted average of a dependent variable of two consecutive time steps by linear interpolation of the values of the variable at the two time steps such as

$$
\theta_{j}=\delta \theta_{j}^{n+1}+(1-\delta) \theta_{j}^{n}
$$

The time derivatives $\dot{\theta}_{j}$ are replaced by Forward finite difference formula such as

$$
\dot{\theta}_{j}=\frac{\theta_{j}^{n+1}-\theta_{j}^{n}}{k}
$$

In view of (19) and (20), equation (17) written as,

$[\mathrm{A}+\delta \mathrm{k}(\mathrm{B}+\mathrm{C})] \phi^{(\mathrm{n}+1)}=[\mathrm{A}-(1-\delta) \mathrm{k}(\mathrm{B}+\mathrm{C})] \phi^{(\mathrm{n})}$

Where, $\boldsymbol{\delta}=\mathbf{1} / \mathbf{2}$ and $\mathrm{n}=0,1,2$,

For a uniform mesh of $\mathrm{N}$ elements, by eq. (18), the global equation (21) takes the form, 


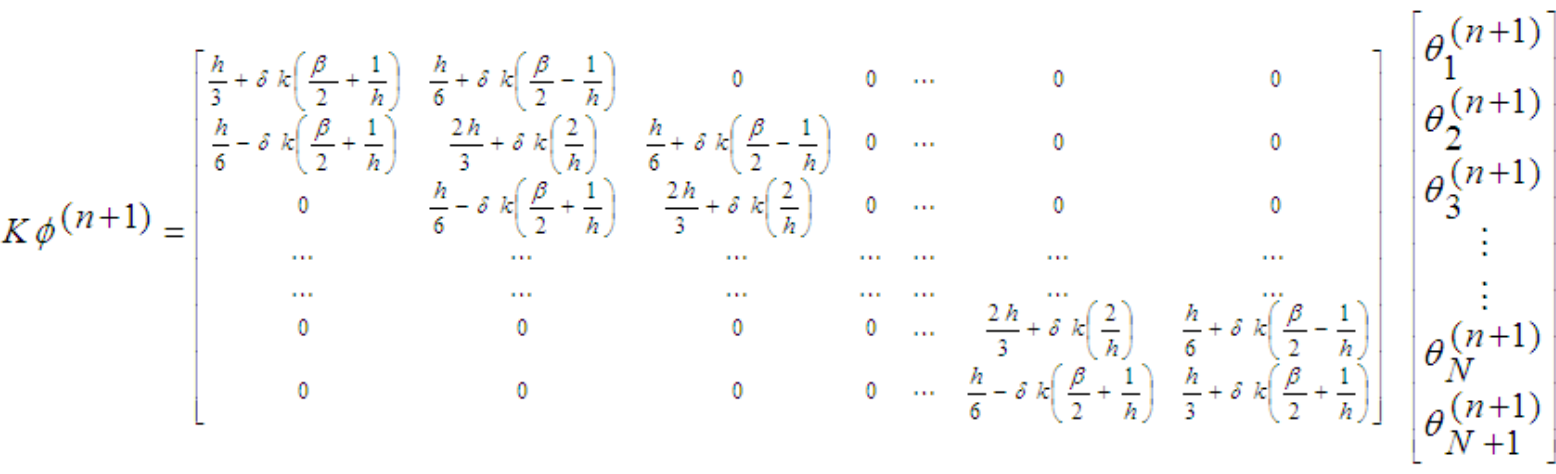

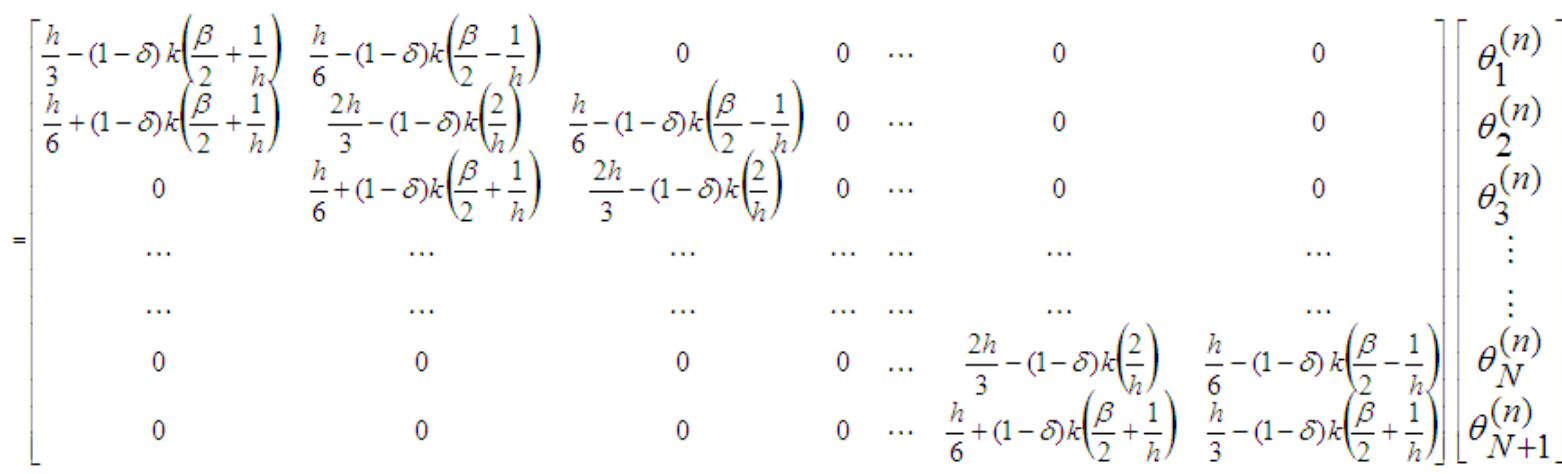

$$
\begin{aligned}
& =\left[\begin{array}{c}
\frac{h}{3}-(1-\bar{\delta}) k\left(\frac{\beta}{2}+\frac{1}{h}\right) \theta_{1}^{(n)}+\frac{h}{6}-(1-\delta) k\left(\frac{\beta}{2}-\frac{1}{h}\right) \theta_{2}^{(n)} \\
\frac{h}{6}+(1-\delta) k\left(\frac{\beta}{2}+\frac{1}{h}\right) \theta_{1}^{(n)}+\frac{2 h}{3}-(1-\delta) k\left(\frac{2}{h}\right) \theta_{2}^{(n)}+\frac{h}{6}-(1-\bar{c}) k\left(\frac{\beta}{2}-\frac{1}{h}\right) \theta_{3}^{(n)} \\
\frac{h}{6}+(1-\bar{\delta}) k\left(\frac{\beta}{2}+\frac{1}{h}\right) \theta_{2}^{(n)}+\frac{2 h}{3}-(1-\bar{\delta}) k\left(\frac{2}{h}\right) \theta_{3}^{(n)} \\
\vdots \\
\frac{2 h}{3}-(1-\delta) k\left(\frac{2}{h}\right) \theta_{N}^{(n)}+\frac{h}{6}-(1-\delta) k\left(\frac{\beta}{2}-\frac{1}{h}\right) \theta_{N+1}^{(n)} \\
\frac{h}{6}+(1-\delta) k\left(\frac{\beta}{2}+\frac{1}{h}\right) \theta_{N}^{(n)}+\frac{h}{3}-(1-\delta) k\left(\frac{\beta}{2}+\frac{1}{h}\right) \theta_{N+1}^{(n)}
\end{array}\right]=F
\end{aligned}
$$$$
\text { Therefore, } K \phi^{(n+1)}=F
$$

Where $\mathrm{K}$ is called global stiffness matrix and $\mathrm{F}$ is called global generalized force vector and $\mathrm{N}+1$ is total number of global nodes.

\subsection{Imposing Boundary Conditions}

We now apply the boundary condition (8) to the global equation (22) of the problem. The boundary condition $\theta(0, \mathrm{~T})=\theta_{0}$ states that $\theta_{1}{ }^{(\mathrm{n}+1)}=\theta_{0}$ for all $\mathrm{n} \geq 0$. Therefore we replace all the entries of $1^{\text {st }}$ row of matrix $\mathrm{K}$ by zero except the diagonal entry $\mathrm{K}_{11}$ replaced by one and replace $1^{\text {st }}$ row of matrix $\mathrm{F}$ by $\theta_{0}$. Thus, by applying boundary condition (8) to equation (22) and simplifying, we get $K \phi^{(n+1)}=F$

Where $\mathbf{K}=$ 


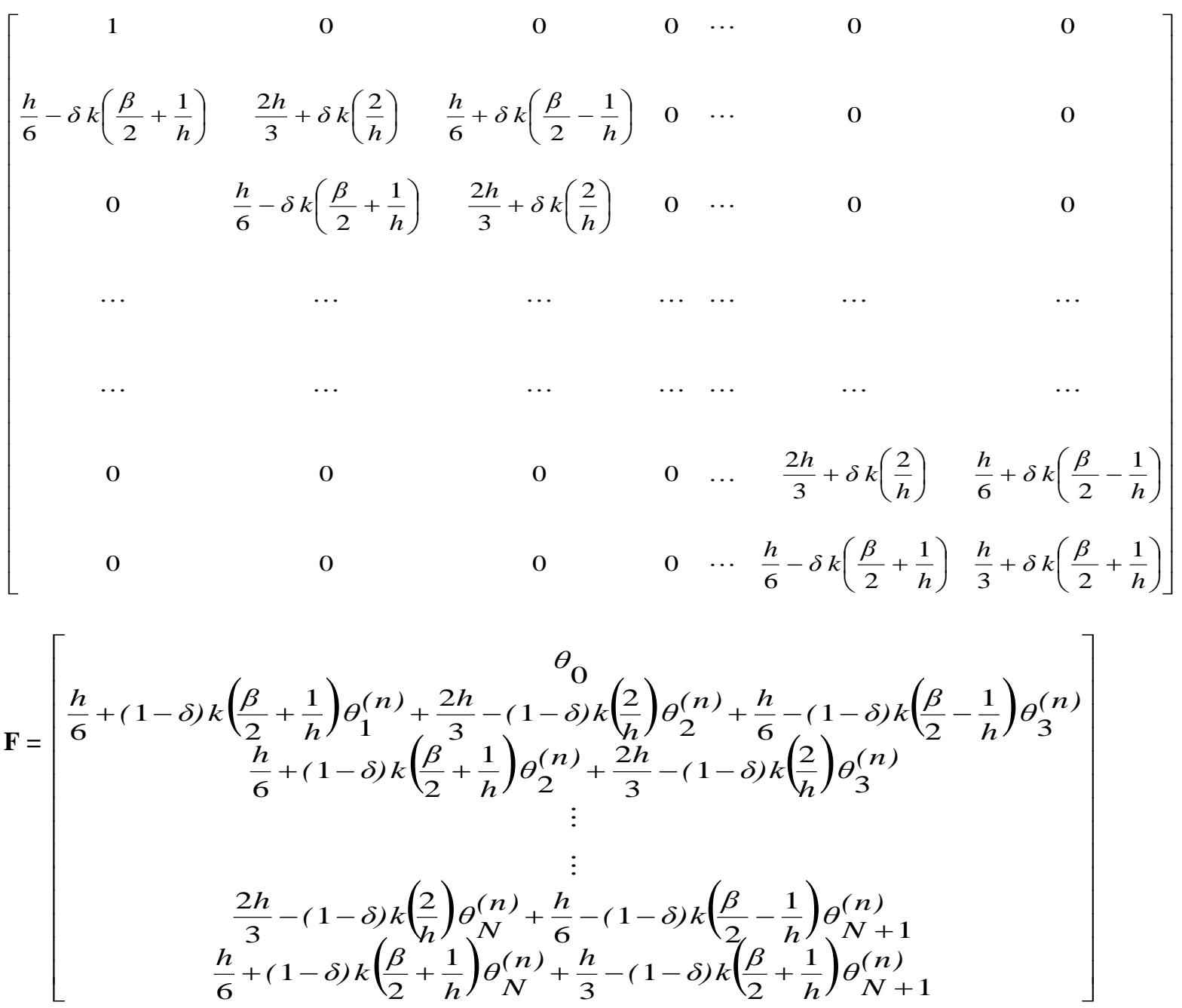

Thus, equation (23) is the resulting system of linear algebraic equation.

\subsection{Solution Of Algebraic Equation}

Solution at the $(n+1)^{\text {th }}$ iteration can be obtained by solving equation (23) using Gauss Elimination Method. At the beginning of the iteration (i.e. $\mathrm{n}=0$ ), we assume the solution $\phi^{(0)}$ from initial condition (9) which requires to have $\theta_{1}{ }^{(0)}=\theta_{2}^{(0)}=$ $=\theta_{\mathrm{N}+1}{ }^{(0)}=0$.

\subsection{Graphical Representation And Interpretation}

The values of theta at time $\mathrm{t}=0.1, \mathrm{t}=0.2, \mathrm{t}=0.3, \mathrm{t}=0.4$ and $\mathrm{t}=0.5$ seconds are

$\begin{array}{lllll}0.5000 & 0.5000 & 0.5000 & 0.5000 & 0.5000 \\ 0.4736 & 0.4985 & 0.5067 & 0.5095 & 0.5104 \\ 0.4454 & 0.4974 & 0.5147 & 0.5205 & 0.5224 \\ 0.4173 & 0.4976 & 0.5242 & 0.5332 & 0.5362 \\ 0.3914 & 0.4998 & 0.5359 & 0.5480 & 0.5520 \\ 0.3704 & 0.5052 & 0.5502 & 0.5652 & 0.5703 \\ 0.3569 & 0.5149 & 0.5677 & 0.5854 & 0.5913 \\ 0.3538 & 0.5301 & 0.5892 & 0.6090 & 0.6156 \\ 0.3640 & 0.5521 & 0.6153 & 0.6365 & 0.6436 \\ 0.3902 & 0.5822 & 0.6469 & 0.6686 & 0.6758 \\ 0.4351 & 0.6218 & 0.6848 & 0.7059 & 0.7130 \\ 0.5010 & 0.6720 & 0.7298 & 0.7492 & 0.7557 \\ 0.5895 & 0.7340 & 0.7829 & 0.7993 & 0.8048 \\ 0.7019 & 0.8087 & 0.8449 & 0.8571 & 0.8611 \\ 0.8388 & 0.8972 & 0.9170 & 0.9236 & 0.9258 \\ 1.0000 & 1.0000 & 1.0000 & 1.0000 & 1.0000\end{array}$




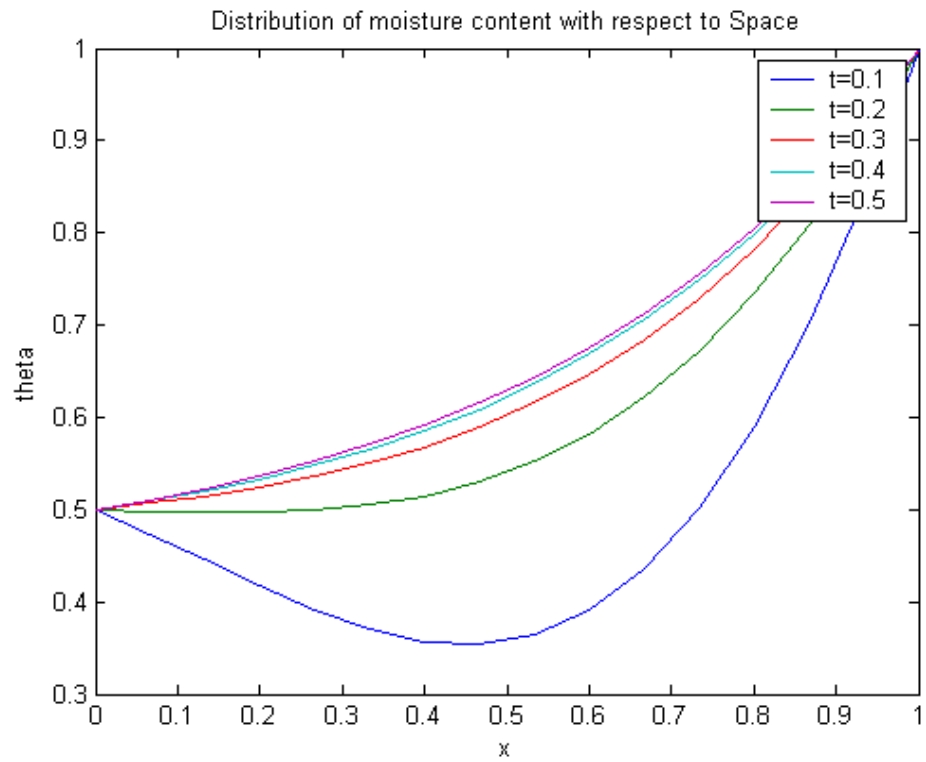

Figure 2(a)

In above graphs, $\mathrm{X}$-axis represents moisture content $(\theta)$ of unsaturated porous media in large basin of length one. $Y$ - axis represents the time ' $t$ ' in seconds Solution obtained with $\beta=\mathbf{b}=\mathbf{2 . 0 3 5}, \quad \boldsymbol{\theta}_{\mathbf{0}}=\mathbf{0 . 5}, \mathbf{h}=\mathbf{1 / 1 5}$ and $\mathbf{k}=\mathbf{0 . 0 0 2 2 2 3}$ for $\mathbf{2 2 5}$ time levels. We consider that the sides of basin are limited by rigid boundaries \& bottom by a thick layer of water table so that water flows only in positive $\mathrm{x}$-direction.

It is clear from graph that $\theta=\theta_{0}(=0.5)$ at layer $\mathrm{x}=0$ and at water table $(\mathrm{x}=1)$, it is assumed to remain $100 \%$ throughout the process of investigation. It interpreted from graphs that as time increases, the moisture content also increases but the rate at which moisture content rises at each point in basin slow down with increase in time.

Now the value of theta at $\mathrm{x}=0.2, \mathrm{x}=0.4, \mathrm{x}=0.6, \mathrm{x}=0.8$ with time $\mathrm{t}=0.1, \mathrm{t}=0.2, \mathrm{t}=0.3, \mathrm{t}=0.4$ and $\mathrm{t}=0.5$ seconds are

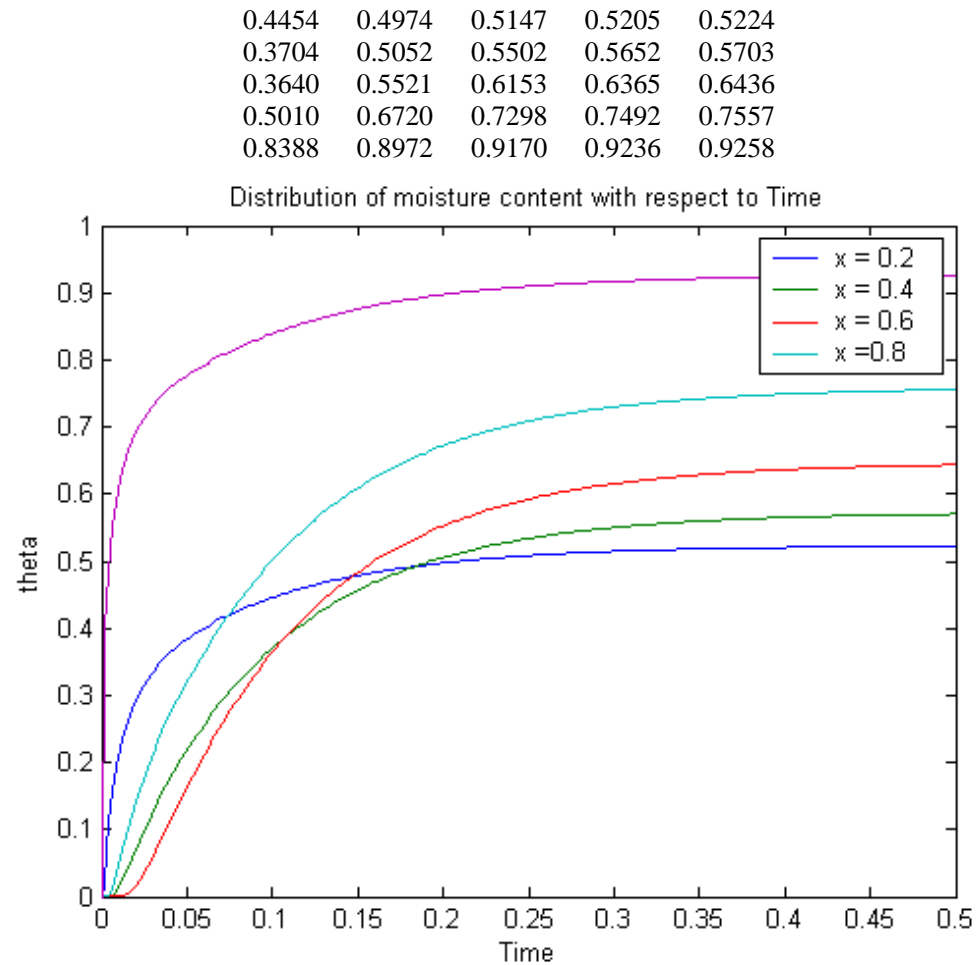

Figure 2(b) 
In above graphs, $\mathrm{X}$-axis represents the time ' $\mathrm{t}$ ' in seconds. $\mathrm{Y}$ - axis represents the moisture content $(\theta)$ of media in large basin of length one.

It is clear from graph that $\theta=0$ initially throughout the region. It interpreted from graphs that as time increases, the moisture content also increases at each point in the basin but the rate at which moisture content rises at each point in basin slows down with increase in time and after some time, it become constant. Also, the rate of increase of moisture content at particular time is decreased as we go towards water table in basin.

\section{Conclusion}

Engineers in several fields have to learn the mechanism of drainage and to apply to problems of water supply, land reclamation and stabilization of foundations and sub grade, and also to the fields of petroleum production and agriculture.

Drainage in general is any provision for the removal of excess water. The common objective of land projects to prevent or eliminate either water logging or inundation or otherwise productive land. Drainage of projected land refers principally to the disposal of surplus natural water adversely affecting irrigation. Practically every area where irrigation has been carried on for time has been affected by high water table. Therefore provision for adequate drainage is an essential part of planning, construction and operation of an irrigation project.

For agriculture purpose, the continued presence of water in excess of that needed for vegetation is harmful. Prolonged saturation of soil excludes air essentially for healthy plant growth and the soil becomes cold, sour and unproductive. Consequently unsaturated or irrigated soils is a necessary evil, so to this type of drainage where originally saturation conditions are existing up to the top.

\section{References}

[1]. Scheidegger, A. E. Physics of flow through porous media, (Revised Ed.). Univ. Toronto P., 1974

[2]. Greenskorn, R.A. Flow phenomena in porous media, Marcel Dekkar Inc., Newyork, 1983.

[3]. Anderson, Computational Fluid Dynamics.

[4]. Chung T.J., Computational Fluid Dynamics.

[5]. Jain M.K., Numerical solution of differential equations. $2^{\text {nd }}$ edition, Wiley Eastern, 1984

[6]. Jain M.K., Iyengar S.R., Jain R.K., Computational Methods for Partial Differential Equations, Wiley Eastern.

[7]. Reddy J.N., An introduction to the finite element method, $3^{\text {rd }}$ edition, McGraw-Hill, Inc., Newyork, 2006.

[8]. Pratap Rudra, Getting started with MATLAB, Version 6, New York Oxford University press,2004

[9]. Chapman J Stephen, Matlab programming for Engineers, $3^{\text {rd }}$ Edition ,Thomson Learning.

[10]. Pradhan V.H., Thesis, A Numerical study of fluid flow problems through porous media.1995.

[11]. Darcy, H., Les Fontains publiques de La Ville Dison Paris, 1856.

[12]. Brownscombe, E.R. and Dyes, A.B., Amer. Petrol Inst. Drill. Prod. Pract. , P. 383

[13]. Enright, R.J., Oil gas J, 53,954,104.

[14]. Graham, J.W. and Richardson, J.G., J. Petrol. Tech., 11, 65

[15]. Rijik, V.M., Izv. Akad. Nauk. SSSR, Utd. Tech. Nauk Mekhan Mashinostor, 2

[16]. Fox, L., Numerical Solution of ordinary and partial differential equation, Macmillan (paraganon), NewYork, 1962.

[17]. Verma, A.P., (1969): The Laplace Transform solution of a one dimensional ground water recharge by spreading, Annali Di Geofisica, 22, 1, p. 25.

[18]. Klute, A., (1952): A numerical method for solving the flow equation for water in unsaturated materials, Sci. 73, 105-116

[19]. Richards, L.A., (1949): Methods of measuring soil moisture tension, Soil Sci., 68, pp. 95-112.

[20]. Richards, L.A., (1953): Water conducting and retaing properties of soil in relation to irrigation, Desert Research res. Council, Israel, Spec. Pub. 2, pp. 523-46.

[21]. Childs, E.C. and Collis- Geroge N., (1950): The Control of soil water, Advan. Argon 2, pp. 233-72

[22]. Croney, D, et al., (1952): The Solutions of moisture held in soil and other porous materials, Road, Res, Tech., Paper 24

[23]. Miller, E.E., and Klute, A., (1967): The dynamics of soil water Part-1, Mechanical forces in irrigation of Agricultural land, Am. Soc. Argon. Madison, Wisconsin, pp. 209-44.

[24]. Muskat, M. Physical Principles of oil production, McGraw Hill, 1949

[25]. Muskat, M., Physics of homogenous fluid, 5, 51, 1954.

[26]. Sharma S.V.K., Problem of partially saturated unsteady state of flow through porous media with special references to groundwater recharge by spreading, J. Sci. Engg. Research, IX, 1, 69, 1965.

[27]. Whiteman, J., (1973): The mathematics of Finite Elements and Applications, Academic Press, New York.

[28]. Reddy, J.N., Applied Functional Analysis and Variational Methods in Engineering, McGraw-Hill, NewYork, 1986; Krieger, Melbourne, FL, 1991.

[29]. Reddy, J.N.,An Introduction to Nonlinear Finite Element Analysis, Oxford University Press, Oxford, UK,2004.

[30]. Smith, G.D. Numerical Solution of Partial Differential Equations, Oxford University Press, 1966.

[31]. Zlamal,M. "Finite Element methods for parabolic equations", Lecture notes in Math., 363, Springer-Verlag,1974. 